\title{
Reassessing logical hylomorphism and the demarcation of logical constants
}

\author{
Catarina Dutilh Novaes
}

Received: 2 November 2009 / Accepted: 6 October 2010 / Published online: 22 October 2010

(C) The Author(s) 2010. This article is published with open access at Springerlink.com

\begin{abstract}
The paper investigates the propriety of applying the form versus matter distinction to arguments and to logic in general. Its main point is that many of the currently pervasive views on form and matter with respect to logic rest on several substantive and even contentious assumptions which are nevertheless uncritically accepted. Indeed, many of the issues raised by the application of this distinction to arguments seem to be related to a questionable combination of different presuppositions and expectations; this holds in particular of the vexed issue of demarcating the class of logical constants. I begin with a characterization of currently widespread views on form and matter in logic, which I refer to as 'logical hylomorphism as we know it'-LHAWKI, for short-and argue that the hylomorphism underlying LHAWKI is mereological. Next, I sketch an overview of the historical developments leading from Aristotelian, non-mereological metaphysical hylomorphism to mereological logical hylomorphism (LHAWKI). I conclude with a reassessment of the prospects for the combination of hylomorphism and logic, arguing in particular that LHAWKI is not the only and certainly not the most suitable version of logical hylomorphism. In particular, this implies that the project of demarcating the class of logical constants as a means to define the scope and nature of logic rests on highly problematic assumptions.
\end{abstract}

Keywords Hylomorphism - Demarcation of logic · Logical constants · History of logic

In this paper, I investigate the propriety of applications of the form versus matter distinction —originally introduced by Aristotle in (meta)physical contexts—-to arguments and to logic in general. The main point I will argue for is that many of the currently

C. Dutilh Novaes ( $\varangle)$

Department of Philosophy, Institute for Logic, Language and Computation (ILLC),

University of Amsterdam, Oude Turfmarkt 141-147, 1012 GC, Amsterdam, The Netherlands

e-mail: c.dutilhnovaes@uva.nl 
pervasive views on form and matter regarding logic rest on several substantive and even contentious assumptions which are nevertheless uncritically accepted. Indeed, many of the issues raised by the application of this distinction to arguments seem to be related to a questionable combination of different presuppositions and expectations. This holds in particular of the vexed issue of demarcating the class of logical constants; much of the literature of the last decades in the field has been devoted to this topic, but it remains essentially an open problem. ${ }^{1}$

In order to understand how and why we came to hold the currently received views on form and matter in logic, it will prove useful to examine the history of applications of the distinction to arguments, yielding what could be described as the thesis of 'logical hylomorphism' (a term coined by J. MacFarlane) ${ }^{2}$ Logical hylomorphism as we know $i t^{3}$ - henceforth, LHAWKI-will be discussed in Sect. 1, but for a first approximation one could say the following: LHAWKI is the doctrine according to which the form versus matter distinction is to be applied to objects such as arguments ${ }^{4}$ so as to outline what is distinctively logical about them-which is associated to their formal aspects - as opposed to their merely material aspects. Thus seen, the form versus matter distinction has the crucial responsibility of demarcating what is logical from what is not logical (in particular, of defining the class of logical constants), and of grounding the validity of valid arguments. In fact, it seems reasonable to say that most of the currently pervasive conceptions of logic rest fundamentally on the particular interpretation of the form versus matter distinction underlying LHAWKI. It is usually in this sense that the slogan 'logic is formal' is put forward, that is, in the sense that logic deals (exclusively) with forms of arguments.

The paper proceeds as follows: Sect 1 presents a characterization of LHAWKI as based on a mereological ${ }^{5}$ form of hylomorphism, and suggests that many of the issues that arise in connection with LHAWKI's account of the validity of arguments and of logic in general stem from this particular application of the form versus matter

\footnotetext{
1 See MacFarlane (2009) for an overview of these discussions as well as a thorough list of references. Gomez-Torrente (2002), van Benthem (1989) and McCarthy (1981) are particularly worth mentioning. MacFarlane (2009) classifies the different positions with respect to the problem of logical constants into four kinds: "the Demarcater, the Debunker, the Deflater, and the Relativist." He also recognizes that the issue is unsettled and that "there is little philosophical consensus about the basis for the distinction between logical and nonlogical expressions". According to this taxonomy, the views I will be defending here are probably best described as a belonging to the Debunker camp. As will become clear, I endorse one of Debunker's main theses: "logic is concerned with validity simpliciter, not just validity that holds in virtue of a limited set of 'logical forms."”

2 MacFarlane (2000) refers to it as a 'tradition' rather than a doctrine, as he correctly notices that there are different notions of form and matter in play, and thus that logical hylomorphism is not a unified doctrine. Nevertheless, as here I will be mainly speaking of the (in MacFarlane's terms) 'schematic' notion of formality, I will be dealing with a specific thesis/doctrine. As such, the term 'formal' is quite ambiguous, allowing for different interpretations, so it is important to bear in mind that here I am concerned with schematic formality.

3 The qualification 'as we know it' is crucial, as it stresses the difference between the form of logical hylomorphism that is now widely (but often tacitly) held and the other forms of logical hylomorphism which could be articulated taking a different form of hylomorphism as their starting point.

4 In this tradition, there is also considerable interest in the logical form of sentences, but for reasons of space I shall here focus on arguments.

5 The mereological terminology will be explained in due course.
} 
distinction to arguments. Section 2 reviews the historical developments that led from Aristotelian, non-mereological metaphysical hylomorphism to mereological logical hylomorphism, LHAWKI. The significance of this historical overview is to show that the particular view on the validity of arguments as related to their form (on a specific understanding of 'form') is the product of a historical (contingent) course of events, and thus not necessarily constitutive of logic as such. Finally, in Sect. 3 I briefly discuss the prospects for the combination of hylomorphism and logic. I argue that there seem to be at least three reasonable options: to let go of hylomorphism entirely with respect to logic; to hold an analogical/metaphorical interpretation of hylomorphism in logic; to take a different version of metaphysical hylomorphism as a starting point, for instance the non-mereological functionalist hylomorphism formulated by Aristotle himself. $^{6}$ At any rate, I conclude that LHAWKI is best abandoned, unless it is able to give a satisfactory answer to the key issues it seems to be neglecting.

\section{Logical hylomorphism as we know it}

Although the thesis that logic is formal is often seen as the traditional, millennia-old account of what is distinctive about logic, MacFarlane's (2000) historical analysis convincingly shows that this is not the case; according to him, it was Kant who first offered a worked-out view of formality as a criterion for logicality. ${ }^{7}$ Before Kant, logic was thought to deal with forms of arguments, to be sure, but not exclusively with forms of arguments. At the same time, the very distinction between the formal and the material aspects of arguments had been available for most of the post-Aristotelian logical tradition broadly construed (ranging from the Ancient Commentators to Scholasticism). The turning point introduced by Kant was to use the form versus matter distinction as a criterion for what is to count as logic. ${ }^{8}$ We shall look into these historical developments in Sect. 2, but first let me offer a characterization of what could be called the classical or 'textbook' view of logic, outlining how crucially it relies on LHAWKI and thus on a particular interpretation of the form versus matter distinction as a demarcating criterion.

In an attempt to circumvent the risk of building a straw-man when characterizing the classical view, let me rely on one of the best introductions to the philosophy of logic available, namely S. Read's Thinking about Logic (1995). It is in the chapter on logical consequence that Read spells out in detail (and then goes on to criticize) the

\footnotetext{
6 Indeed, the form of hylomorphism underlying LHAWKI is significantly different from Aristotle's own hylomorphism, even though the latter is clearly the indirect historical source of the former. Describing the different stages of this transformation is one of the purposes of the present contribution.

7 But what Kant means by 'formal' is not exactly what is now typically meant when it is said that logic is formal (although traces of Kantian idealism are still to be found in current conceptions of logic, e.g. the idea that logic is completely divorced from the empirical world). This is significant, as it implies that the criticism being developed here does not extend to a Kantian demarcational project such as MacFarlane's (even though I believe that there are equally serious problems with the Kantian approach).

8 Although Kant usually speaks of the form versus content opposition rather than the form versus matter opposition, in particular in the first Critique.
} 
extent to which the classical view is intertwined with a particular application of the form versus matter distinction to arguments. ${ }^{9}$

Speaking very generally, we could say that one of the goals of logic is to establish effective methods to differentiate valid from invalid arguments in a systematic way; after all, once one is in possession of such a method, one can adjudicate for arguments in general whether they are valid, and in particular whether knowledge of the premises entitles one to knowledge of the conclusion. Now, the form versus matter distinction as applied to arguments promises to deliver just that. The basic idea is that, in an argument, there is something that corresponds to its form and something that corresponds to its matter; moreover, the validity of an argument is viewed as a property exclusively related to its form.

First, on the classical view, validity is a matter of form. Individual arguments are valid only in virtue of instantiating valid logical forms; one proposition is a logical consequence of others only if there is a valid pattern which the propositions together match. (Read 1995, 36)

So on this view, the form of an argument is precisely that in virtue of which it is valid. There is a class of argument-forms which are deemed to generate valid arguments, and the validity of a particular argument depends solely on its displaying one of these forms: nothing else is either required or allowed to ground the validity of an argument. Thus seen, it seems natural that logic must deal exclusively with forms, if it is to be a method to distinguish valid from invalid arguments.

So validity is a matter of form, and the task of logic is to provide techniques for identifying and discerning the logical form of various arguments, and for determining whether the forms discovered in this way are indeed valid. (Read 1995, 37)

One could of course wonder what exactly is being captured by means of the focus on forms. How does one establish that a given argument-form yields valid arguments in the first place? In fact, the first question to be asked is: what is so special about valid arguments anyway? A few lines above I have already hinted at what is at stake when I said that, in a valid argument, knowledge of the premises entitles one to knowledge of the conclusion. The point is of course that a valid argument is expected to expand our initial state of knowledge: if things are as described by the premises, then surely they are also as described by the conclusion (provided that the argument is valid). Hence, on the basis of a valid argument, we come to know more about whatever objects the argument is about. For this to occur, valid arguments must be truth-preserving, and valid forms are those whose instantiations are all truth-preserving.

According to the classical account, [the criterion of validity for arguments and argument-forms] is truth preservation. That is, an argument-form is valid if,

\footnotetext{
9 As described here, the classical view is essentially a portrait of MacFarlane's Demarcater, but a Relativist and a Deflater may also be partisans of the classical view if they hold that logic deals exclusively with forms of arguments and with formal validity.
} 
however the schematic letters are interpreted, the result does not consist of a collection of true premises and a false conclusion. (Read 1995, 37)

There is an apparent tension between the somewhat conflicting ideas of arguments being valid solely in virtue of their form as opposed to their being valid in virtue of the property of truth-preservation. ${ }^{10}$ This tension is diagnosed by Etchemendy (1990) in terms of two competing notions of logical consequence: in his terminology, the representational notion - truth-preservation under modal variation-and the interpretational notion-validity ensured by the form of an argument, a form which can generate several substitutional instances. What is then the ultimate ground for the validity of an argument, form or truth-preservation?

A sensible solution to this tension follows the general idea of reflective equilibrium as first described by Goodman $(1955,64) .{ }^{11}$ The idea is that we intuitively subscribe to a class of arguments as being valid, roughly those that clearly guarantee truth-preservation; the task of logic is then to capture patterns among these valid arguments so as to facilitate the recognition of the validity or invalidity of other arguments, i.e. those which are neither obviously valid nor obviously invalid. On this picture, whenever the (logical) theory deems an intuitively invalid argument as valid, or the converse, adjustments are required: either the theory needs to be changed, or the original intuitions must be corrected (whence reflective equilibrium). Thus seen, truthpreservation and formal validity need not be in tension with one another-they are in fact two sides of the same coin; it is possible to capture and ensure truth-preservation by means of (exclusive) focus on forms on a theoretical level.

But what exactly is the form of an argument? ${ }^{12}$ How do we obtain the separation of form from matter, given a particular argument? This is indeed the Achilles' heel of the whole account, as we shall see. The general procedure is the following:

First, note that as presently outlined, truth-preservation is essentially a substitutional criterion. We take an argument, M. We replace a certain amount of terminology in $\mathbf{M}$ by schematic letters, to obtain an argument-form, $\mathbf{M}^{\prime}$. We then interpret the schematic letters in $\mathrm{M}^{\prime}$ in various ways, looking to see whether any instance of $\mathbf{M}^{\prime}$ has true premises and false conclusion. (Read 1995, 40)

If we encounter one instance of $\mathrm{M}^{\prime}$ which is clearly invalid (true premises but false conclusion), then we can deem $\mathbf{M}^{\prime}$ to be an invalid argument-form. A valid argumentform must yield nothing but instances satisfying the truth-preservation criterion. This of course does not mean that every instance of $\mathrm{M}^{\prime}$ (the invalid argument-form) will have true premises and a false conclusion; but an instance of $\mathbf{M}^{\prime}$ having either false

\footnotetext{
10 On the classical account, truth-preservation is a necessary but not sufficient criterion for (formal) validity.

11 The term 'reflective equilibrium' itself was introduced only later on, by Rawls (1971).

12 This question can also be seen as pertaining to the metaphysical status of the forms of arguments. What kind of entities are they? Is there one unique form for each argument? How is the form of an argument related to its grammatical structure? The metaphysics of arguments and forms of arguments tends to receive scarce attention in the literature, and this is precisely one of the shortcomings of LHAWKI.
} 
premises or a true conclusion is not going to be a valid argument, as it is not valid in virtue of its form. ${ }^{13}$

Thus seen, the form versus matter distinction applied to arguments relies crucially on a partition of the vocabulary: some of the terms of an argument are thought to pertain to its form, while others are thought to pertain to its matter. Those that are replaced by schematic letters pertain to its matter, and those that are allowed to remain fixed pertain to its form. Naturally, besides the partition of the vocabulary, the particular disposition of the terms pertaining to the form of an argument is also constitutive of this form. Otherwise, arguments having the same terms that remain fixed but in a different configuration would have the same form, which is of course not what the LHAWKI tradition holds to be the case. ${ }^{14}$

Even though those who tacitly accept LHAWKI hardly ever discuss the exact metaphysical status of the form and matter of arguments thus described, if pressed they might say something like this: the form of an argument corresponds to (or is represented by) the schema generated by the uniform substitution of some of its terms by schematic letters, and its matter corresponds to the terms having been replaced. ${ }^{15}$ Thus, some structural information also belongs to the form of an argument, but the truly thorny issue is how to demarcate the terminology in $M$ to be replaced from the terminology which is to remain fixed in order to separate the form from the matter of an argument. If the point is to offer a principled criterion for this partition (as it should be, if the partition is intended to be the means by which logic is demarcated from other disciplines), then the endeavor is everything but straightforward, as spelled out in the following passage:

This raises an immediate problem, of course: which substitutions are permissible - that is, which terms may be replaced? For the classical account does not permit every term in an argument to be open to substitution. This restriction is contained within the notion of form, of which we have perhaps said too little. Note that in all the forms above [examples omitted], one word was not replaced by a schematic letter, namely, the word 'all'. This is, on the classical conception (and indeed all others) a reserved term, part of the logical vocabulary. In exhibiting the logical form of an argument, we replace all expressions other than those in the logical vocabulary by schematic letters. Logical words include 'all', 'some', 'if', 'and', 'or', 'not', and a number of others. Indeed, some words are treated sometimes as logical, sometimes not, yielding different logics. For example, if 'necessarily' is treated as a logical word, we obtain modal logic, an extension of classical logic;

\footnotetext{
13 Etchemendy $(1990,2008)$ claims that the view according to which truth-preservation in all substitutional instances is the proper criterion of validity for arguments and argument-schemata makes the mistake of "confusing the symptoms of logical consequence with their cause" (Etchemendy 2008, 264). The idea is that, if what is required to establish whether a given argument is valid is that all its substitutional instances be truth-preserving, then one is already required to know whether this particular argument (being one of its substitutional instances) is valid, which is precisely what one seeks to establish. For Etchemendy, truth-preservation is a symptom (consequence) of validity, not its cause.

14 Obviously, 'Not every man is an animal' and 'Every dog is not a stone' share the same logical terminology but not the same logical form.

15 John Corcoran is one of the few to have inquired into the metaphysics of schemata-see Corcoran (2008).
} 
if not, non-modal, that is, standard logic. If the 'is' of identity [...] is taken as a logical term, we obtain classical logic with identity, if not, not. Many extensions of classical logic (so themselves essentially classical) are obtained by extending the logical vocabulary. (Read 1995, 40/41)

Thus seen, it is clear that, from this point of view, the logical versus non-logical distinction neatly corresponds to the form versus matter distinction (on a particular interpretation of it). The assumption warranting this correspondence is that logic deals exclusively with forms, namely forms of arguments, and thus that to be logical and to be formal are equivalent notions. The form of an argument corresponds to a particular subset of its lexicon in a particular disposition, and precisely this subset is thought to correspond to the logical constants being used in the argument. All the rest is material-or equivalently, non-logical.

In his 'On the concept of following logically' (1936/2002), which is a locus classicus for the (recent) development of the standard views I am portraying here, Tarski recognizes that the logical versus non-logical partition is the cornerstone of his account: "At the foundation of our whole construction lies the division of all terms of a language into logical and extra-logical" (Tarski 1936/2002, 188). But the article ends with Tarski revealing his own skepticism on the prospects for such a partition:

\section{[3.3 Prospects] [3.3.1] Clearly, further investigations may throw a lot of light} on the question which interests us; perhaps one will succeed with the help of some weighty arguments of an objective character in justifying the dividing line traced by tradition between logical and extra-logical terms. [3.3.2] Personally I would not be surprised however even if the result of these investigations were to be decidedly negative and if hence it would turn out to be necessary to treat such concepts as following logically, analytic sentence or tautology as relative concepts which must be related to a definite but more or less arbitrary division of the terms of a language into logical and extra-logical [...]. (Tarski 1936/2002, 189)

A few decades later, Tarski returned to this issue, but this time more optimistic about the prospects of formulating a principled criterion for the distinction between logical and non-logical terms (or more generally, notions, which are for Tarski non-linguistic objects). In a 1966 lecture (published posthumously in 1986), Tarski proposed a version of the notion of permutation invariance as a criterion for a principled distinction. ${ }^{16}$ On this account, truth-functional connectives, quantifiers and binary relations such as identity and diversity are ruled-in as logical, which is prima facie a desirable result. Indeed, permutation invariance (under different formulations) is still widely viewed as possibly the best candidate for a principled criterion for logicality. ${ }^{17}$ But it does not seem to do justice to many of the important developments in logic of the last decades, ruling out as non-logical many terms and notions that we would be prepared to call logical (at least within a given logic). For example, if interpreted on Kripke-frames, modal notions would be permutation-invariant (and thus logical) only

\footnotetext{
16 Simons (1992) offers an illuminating account of Tarski's transformation from Skeptic to Demarcater.

17 See in particular the work of G. Sher and of D. Bonnay.
} 
if interpreted on: (i) frames where every possible world is related to every possible world, (ii) frames with empty accessibility relation, or (iii) frames with only the reflexive accessibility relation. ${ }^{18}$ But this is an awkward implication, to say the least: why should a necessity-box operator be logical in such frames, while its S4 counterpart is not? The permutation-invariance criterion also seems to rule-in as logical notions whose logical status is debatable, in particular numerical relations. ${ }^{19}$

The debate is at this point inconclusive; none of the principled criteria that have been proposed so far to justify the logical versus non-logical distinction have met with unanimous acceptance, but at the same time quite a few people still believe in the possibility (or in any case in the crucial importance) of ever encountering such a principled criterion. ${ }^{20}$ Indeed, failure to offer a proper treatment of this issue is obviously a major obstacle for a satisfactory philosophical account of logic based on the form versus matter distinction along the lines of LHAWKI. Of course, we cannot at this point exclude the possibility of ever finding such a principled distinction, but given all the efforts that have already been devoted to this endeavor, it seems to me that the prospects are quite meager. More importantly, the need for a principled criterion seems to emerge from an uncritical application of the form versus matter distinction to arguments and from the attribution of a demarcational (as opposed to merely practical) function to this distinction. Now, if under closer scrutiny it becomes apparent that some of the underlying assumptions are unwarranted, one may feel tempted to conclude (as I do) that the quest for a principled partition of logical from nonlogical expressions is a misguided enterprise.

So let me spell out in more detail the assumptions being made. Lest the reader should still think I am describing a straw-man, here is a passage from a renowned logic textbook, Gamut's Logic, Language and Meaning vol. 1, where the LHAWKI ideology is clearly presented:

We say that (1), (7) and (8) [examples of arguments previously given] have a particular form in common, and that it is this form which is responsible for their

\footnotetext{
18 The idea (proposed in van Benthem 1989, and further discussed in MacFarlane 2000) is to add worlds to the primitive set of types (along with objects and propositions) and to require that logical notions be invariant under the permutation of worlds as well, not only of objects.

19 So a quantifier such as 'there are exactly two things' is ruled-in as logical, even though for there to be two things (in a given domain) seems to be a substantive, non-logical fact. In Etchemendy's terms, one could say that the permutation-invariance criterion seems both to undergenerate and to overgenerate with respect to what one may be prepared to consider a logical constant on intuitive grounds. Interestingly, the fact that the permutation invariance criterion is mainly sensitive to cardinality and quantities had already been noticed by Tarski himself: "it turns out that our logic is even less than a logic of extension, it is a logic of number, of numerical relations" (Tarski 1966/1986, 151). So it seems that the idea of permutation invariance as a criterion for logicality rests on the contentious assumption that logic is fundamentally about numbers and quantities.

20 Such as Sher (2008), Feferman (1999) and Bonnay (2008). MacFarlane, for example, argues that "the permutation invariance criterion does not deliver what it promises" $(2000,175)$, but then proposes a different, equally principled criterion for the demarcation of logic, based on his notion of 1-formality. MacFarlane sees permutation invariance as a necessary though not sufficient criterion for logicality.
} 
validity. This common form may be represented schematically like this:

(11) \begin{tabular}{l}
$\mathrm{A}$ or B \\
Not A \\
\hline $\mathrm{B}$
\end{tabular}

These schematic representations are called argument schemata. The letters A and B stand for arbitrary sentences. Filling in actual sentences for them, we obtain an actual argument. Any such substitution into schema (11) results in a valid argument, which is why (11) is said to be a valid argument schema. (Gamut 1991, 3)

A little further:

Logic, as the science of reasoning, investigates the validity of arguments by investigating the validity of schemata. For argument schemata are abstractions which remove all those elements of concrete arguments which have no bearing on their validity. (Gamut 1991, 4)

Such claims can be found in virtually every introductory textbook to logic, ${ }^{21}$ and are thus the kind of 'philosophical' account of logic that a student is most likely to encounter in her/his first contact with logic. Now, as is well known from studies in the sociology of science, introductory textbooks are extremely powerful in establishing and disseminating scientific ideology. Once such beliefs settle in at an early stage of a student's intellectual development, they tend to become extremely entrenched and are often subsequently uncritically accepted as truisms; indeed, this seems to be the case of LHAWKI, which is (tacitly but widely) presupposed in much of our views and beliefs about logic.

So here is a summary of the main tenets of the LHAWKI position:

1. In every argument, there is something that corresponds to its form and something that corresponds to its matter.

2. The form of an argument is related to ${ }^{22}$ a proper subset of the set of its vocabulary, in a given disposition; the matter of an argument is related to the complement set of the subset corresponding to its form.

3. The form of an argument can be rendered by means of a schema.

4. The form of a valid argument is that in virtue of which it is valid.

5. Given that logic is the systematic study of the validity of arguments, it is essentially concerned with forms of arguments.

\footnotetext{
${ }^{21}$ By taking these passages from Gamut I do not wish to imply that this textbook is defective or 'worse' than others, much to the contrary; the point is precisely that the authors of Gamut express this ideology more clearly than most other textbooks.

${ }^{22}$ I use 'is related to' rather than stronger alternatives such as 'is determined by' in order to make my description of LHAWKI as general as possible. However, I suspect that the stronger alternatives are actually what most people have in mind.
} 
6. Substitutivity is the technique suitable for this systematic study; the terms of an argument pertaining to its matter are substituted to generate instances of an argument-form, while the terms pertaining to its form are allowed to remain fixed.

7. The terms that are allowed to remain fixed (those that pertain to its form) are the logical terms of an argument-also known as logical constants, precisely because they remain fixed-while the terms that undergo variation (which pertain to its matter) are the extra-logical terms of an argument.

8. Logic as a discipline can be demarcated by means of a demarcation of the class of logical constants, given that logic is the study of argument-forms and thus has logical constants as its objects of study.

These are some of the assumptions that seem to underlie strong claims such as:

[T]here is little philosophical consensus about the basis for the distinction between logical and nonlogical expressions. Until this question is resolved, we lack a proper understanding of the scope and nature of logic, and of the significance of the distinction between the "formal" properties and relations logic studies and related but non-formal ones. (MacFarlane 2009, Introduction)

Why is it that only through the distinction between logical and nonlogical expressions can we attain a proper understanding of logic? Couldn't there be other approaches towards a philosophical account of logic? It would seem that such claims are only justified if theses 1-8 are fully endorsed; rejecting any of them might open up the possibility for an alternative philosophical account of logic.

Clearly, the doctrines of LHAWKI are based on the presumed propriety of applying the (originally metaphysical) distinction between form and matter to arguments. However, an analysis of the metaphysics of arguments, which would be required to warrant this assumption, is usually not forthcoming. For starters, when speaking of arguments, what kind of entities are we dealing with exactly? There are quite a few possibilities, such as: argument-types, argument-tokens (both linguistic entities), arguments as mental constructs, arguments as (platonic) abstract entities. Presumably, the ontological status of the form of an argument will be related to the ontological status of an argument itself. Given that LHAWKI typically does not offer an account of the metaphysics of arguments, one may be inclined to offer one on its behalf, and it seems to me that, within this tradition, argument-types are typically viewed as the objects to which the considerations spelled out above apply.

By that as it may, if my characterization of LHAWKI by means of theses 1-8 is correct, then a few considerations on the metaphysical assumptions being made concerning the form versus matter distinction suggest themselves.

(I) The version of hylomorphism underlying LHAWKI is what can be described as a mereological hylomorphism, according to the following definition of mereological hylomorphism: "any account which not only views wholes as compounds of matter (hule) and form (morphe), but which also takes both of these components (and, in particular, form) to be themselves parts, strictly and literally speaking, of the whole they compose" (Koslicki 2006, 717). Non-mereological hylomorphism is in turn a hylomorphic account of entities 
(wholes) according to which form is not a part of the whole. ${ }^{23}$ Insofar as arguments (or argument-types) can be seen as wholes, it would seem that LHAWKI views the forms of arguments as constitutive parts thereof, given that the form of an argument is partially determined by a subset of its vocabulary. Mereological hylomorphism does not require that form and matter have the same ontological status in order for both to constitute parts of the whole ${ }^{24}$; so the fact that, according to LHAWKI, logical constants have a special status does not qualify it as non-mereological. Moreover, the fact that the form of an argument also contains some structural information related to the disposition of the terms does not invalidate the observation that the form of an argument seems to be a constitutive part of the whole. That a (strict) partition of the vocabulary is a necessary (though not sufficient) element to determine the form of an argument is, or so it seems to me, sufficient to justify the claim that the hylomorphism underlying LHAWKI is mereological. (This is particularly conspicuous in theses 2, 3 and 6.)

(II) The partition between the form and the matter of an argument is meant to be unique; equivalently, there is one single form per argument. Of course, in practice this is not always taken very seriously; it is sometimes more convenient to represent an argument taking the sentences involved as its basic constituents (and to analyze it by means of propositional logic, for instance), while at other times it is necessary to spell out the internal structure of the sentences (outlining their predicates, modal terms etc.). But if the form of an argument is that in virtue of which it is valid, and validity is understood in an absolute sense (i.e. not as system-relative), then the implication seems to be that there is exactly one real, actual form per argument, even if different schemata can be seen as (more or less) adequate approximations of it. (This is particularly conspicuous in theses 4, 6 and 7.)

(III) The partition between the form and the matter of an argument is meant to be principled and sharp. Presumably, there is a fundamental, essential difference between form and matter, generally speaking, and thus between the form and matter of arguments in particular; a partition between these two constituents of an argument should reflect the factual, ontic distinction really present in it. That there is a factual basis for the distinction is presupposed in the claim that arguments are valid in virtue of their form and in the idea that a demarcation of the class of logical constants is to yield an adequate demarcation of logic as a discipline. (This is particularly conspicuous in theses 4, 5, 7 and 8.)

In short, the version of hylomorphism underlying LHAWKI seems to be committed to a mereological account of the form-matter compound, to uniqueness of form in each argument and to an essential, factual distinction between form and matter of arguments. Of course, many have found some of these assumptions to be unpalatable.

\footnotetext{
23 Here, I make no use of (or commitment to) other mereological properties of parts and wholes recognized in the literature; my use of the mereological apparatus is rather minimal, and is restricted to what is spelled out in this definition.

24 Even in Koslicki's account of Aristotle's hylomorphism as mereological, form and matter do not have the same ontological status even though they are both, strictly speaking, parts of the mereological whole.
} 
Etchemendy, for example, has criticized the idea of uniqueness of logical form; others have questioned the need for a principled criterion separating logical from extra-logical expressions. Indeed, one can adopt a pragmatist view of the distinction (as discussed in Prior 1976, Wagner 1987 and Warmbrod 1999), according to which the criteria for such a distinction are directly related to the $\operatorname{task}(\mathrm{s})$ at hand, i.e. the particular application(s) that the logical system thus formed is to have. On the pragmatist view, there is no principled, absolute distinction between the form and the matter of an argument; the distinction is stipulated relative to a particular task. ${ }^{25}$ If the goal is to study the logic of modal notions, then modal terms are to be treated as logical constants; if the goal is to investigate epistemic or deontic notions, then epistemic or deontic terms are to be treated as logical constants; and so on and so forth. ${ }^{26}$

But of course, those seeking a principled criterion-in particular, those who think that a great deal is at stake, most importantly a characterization and demarcation of logic as a discipline — cannot be satisfied with this approach; they will require further arguments if they are to be convinced that they should abandon the quest for a principled distinction altogether. Indeed, one of the purposes of the present discussion is precisely to prompt a reflection on the assumptions that underscore the view that a proper understanding of the scope and nature of logic hinges entirely on the possibility of finding a principled demarcation for the class of logical constants. If these assumptions turn out to be unwarranted, then this particular philosophical approach to logic may turn out to be misguided after all.

\section{The history of logical hylomorphism as we know it}

In this section, I sketch the history of applications of the form versus matter distinction to arguments. The idea is to suggest that the particular view on the validity of arguments as related to their form as well as the view that the scope and nature of logic is determined on the basis of the form versus matter distinction are products of a historical (contingent) course of events, and thus not necessarily constitutive of logic as a discipline. The crucial steps in this development were: (1) viewing arguments as objects to which the form versus matter distinction could legitimately be applied; (2) establishing the distinction between the form and the matter of arguments on the basis of a (strict) partition of the vocabulary, yielding mereological logical hylomorphism; (3) using the distinction between the form and the matter of arguments as a means to characterize the very scope and nature of logic.

Contrary to what one might expect, the first applications of the form versus matter distinction to logic, and more specifically to arguments, are not to be found in Aristotle, as J. MacFarlane remarks: "Surprisingly, the father of both formal logic

\footnotetext{
25 This roughly corresponds to MacFarlane's Relativist. But notice that a pragmatic approach to the issue of logical constants need not imply logical pluralism or system-relativism: if one thinks that there is one quintessential task that logic must fulfill (as seems to be the case of Warmbrod, for example), then one may want to maintain that there is a unique set of logical constants that best suits the job in question, but that the criterion is purely pragmatic.

26 This is generally the attitude of practicing logicians; finding a principled demarcation for logic tends to be a worry for the philosopher of logic rather than for the logician.
} 
and hylomorphism was not the father of logical hylomorphism"27 (MacFarlane 2000, 255). Indeed, one might expect that Aristotle himself would have applied the form versus matter distinction to logic and logical objects on the basis of the observation that he is both the first 'formal logician' and the first to develop and apply extensively the form versus matter distinction in other domains such as biology, physics and metaphysics. However, nowhere in the Organon does Aristotle apply the distinction to logical objects such as arguments or sentences. In fact, he applies the form-matter distinction to arguments only twice, in the Metaphysics 1013b19-20 and in an almost identical passage in the Physics 195a18-19. There he observes, without much elaboration (in the context of a discussion of the four causes) that the premises are matter for the conclusion (of an argument), "in the sense of "that from which", (which is a familiar formulation for the notion of material cause). But clearly, this observation does not in any way anticipate the doctrines underlying LHAWKI. And yet, it is undoubtedly the Aristotelian form-matter dichotomy (as opposed to, for instance, Plato's notion of Forms) that is the indirect historical source of these doctrines.

Upon closer inspection, however, it is not so surprising that Aristotle was not the 'father' of logical hylomorphism, and more specifically of logical hylomorphism as we know it-LHAWKI. Firstly, Aristotle did not apply the form versus matter distinction indiscriminately; for him, only special kinds of entities displayed a hylomorphic structure, in particular primary substances. Linguistic objects such as arguments would not straightforwardly qualify to be described as compounds of form and matter. Secondly, Aristotle's own metaphysical hylomorphism appears to be essentially non-mereological: form is not a part, strictly speaking, of the whole. Rather, form is the principle of unity ${ }^{28}$ articulating the different parts of the whole, which constitute its matter. ${ }^{29}$ Thus, even if Aristotle had applied the form versus matter distinction to arguments, most likely the result would have been some form of non-mereological logical hylomorphism, and thus not mereological LHAWKI.

The first step in the radical transformation from Aristotelian non-mereological, metaphysical hylomorphism to mereological LHAWKI took place in the tradition of the later Greek Antiquity, also known as the tradition of the Ancient Commentators, ranging from the second century A.D. to the sixth century A.D. (roughly, from Alexander of Aphrodisias to Ammonius and Boethius). In this tradition, applications of the form versus matter distinction to arguments (syllogisms in particular) are very pervasive, and the two main loci for this application are commentaries on the Prior

\footnotetext{
27 MacFarlane immediately adds in a footnote that it might be "no accident that Aristotle refrains from applying his hylomorphic concepts to logic", relying on M. Burnyeat's claim that Aristotle viewed logic as (strictly) separated from metaphysics and physics.

28 "A principle of unity for a given item is a relation holding of some other items, such that (origins aside) what it is for the given item to be is for the relation to hold among these items" (Johnston 2006, 653).

29 It is true that a mereological reading of Aristotle's own account of hylomorphism has its proponents (Koslicki 2006), but on this matter I side with the more mainstream reading of Aristotle as developing a non-mereological hylomorphism. Koslicki offers compelling arguments for her view, but overall the case for a non-mereological interpretation of Aristotle's hylomorphism seems to me to be stronger.
} 
Analytics and on the Sophistical Refutations, thus primarily concerning arguments (syllogisms). ${ }^{30}$

In Alexander of Aphrodisias' commentary on the Prior Analytics, one finds the oldest still extant applications of the form-matter distinction to arguments. ${ }^{31}$ Here is an exemplary passage:

The [syllogistic] figures are like a sort of common matrix: by fitting matter into them, it is possible to mould the same form in different sorts of matters. For just as things fitted into one and the same matrix differ not in form and figure but in matter, so it is with the syllogistic figures. (Alexander of Aphrodisias, in $\mathrm{Apr}$ 6.16-21, translation p. 48)

Two points are particularly worth noticing in this passage: the mould analogy, and the attribution of the status of form to syllogistic figures. ${ }^{32}$ It is well known that Aristotle uses the mould analogy to clarify the form-matter distinction quite frequently; he says for example that the form of a statue is the shape it receives from the mould, while its matter is the brass out of which it is made. ${ }^{33}$ Thus, starting from Aristotle's own analogy (which induces the notion of form as shape or spatial structure), Alexander (or

\footnotetext{
30 One may speculate that a preliminary step towards viewing arguments as objects to which the form versus matter distinction could legitimately be applied was the different status accorded to logical objects such as arguments by the Stoics. (I owe this point to Arianna Betti.) The Stoics conferred a special place in their ontology to linguistic objects, which they referred to as lekta; thus, one could say that the Stoics had a 'reified' view of arguments which Aristotle did not share. The Ancient Commentators may have been influenced by the Stoic reification of arguments in their applications of the form versus matter distinction to such objects. But at this point, this is no more than speculation, and significantly more historical work would have to be done in order to corroborate this hypothesis. Also, it is worth noticing that the different Commentators did not make a uniform use of these concepts and of the accompanying terminology with respect to logic; in effect, there was not one unique, quintessential logical application of the form-matter distinction (see Barnes 1990, 41; see also Barnes 2007, Chap. 4) as there seems to be one now (the standard view described above).

31 Some scholars think that such an application was probably not Alexander's own innovation, as he "does not give the impression that he is using a terminology of his own invention" (Ebbesen 1981a, 95). But to my mind the evidence is inconclusive either way, and Alexander may just as well have been the first to apply the form versus matter distinction to arguments.

${ }^{32}$ Let me just refresh the reader's memory on some of the details of syllogistic. Aristotle starts with four classes of categorical sentences: universal affirmatives (A), particular affirmatives (I), universal negatives (E) and particular negatives $(\mathrm{O})$. The figures (Aristotle explicitly recognizes three of them, but a fourth one was often considered as well already in the Middle Ages) correspond to the four possible combinations of the three terms composing a syllogism (the two extremes and the middle term) in each of its three sentences. Finally, placing the four kinds of categorical sentences into the four figures yields the different moods - combinatorially, there are 256 possible combinations of sentences, and thus 256 different moods. Of these, 24 are thought to yield valid arguments; see Lagerlund (2004, Sect. 1).

33 See for example Metaphysics VII-8. But Aristotle's uses of artifact examples in order to clarify this distinction are misleading in that they suggest a notion of form (and accordingly, of matter) that is ultimately ineffective when applied to the actually important cases (most notably living beings). As argued by Cohen $(1992,58)$, "the problem with the artifact model is that it oversimplifies hylomorphism and ultimately misrepresents it in the cases that are most important to Aristotle." Given that the artifact analogy appears to have been the starting point for the application of the form-matter distinction to arguments by the Ancient Commentators, and given that it arguably misrepresents the gist of Aristotle's own metaphysical hylomorphism, it is clear that, from the start, this form of logical hylomorphism already parted significantly with Aristotle's version of hylomorphism.
} 
whoever else before him) may have come to the idea of a similar analogy regarding the syllogistic figures. ${ }^{34}$ Indeed, the generation of different specific syllogistic arguments by means of variation of terms while following the same pattern (the same figure) bears some resemblance to the process of using a mould to obtain different statues from different portions of matter. The idea is that different syllogistic arguments belonging to the same figure are different from each other in the same way that statues made with the same mould are different from each other.

Moreover, Aristotle's consistent use of schematic letters as place-holders in both Analytics clearly suggests that what undergoes variation are the terms that are replaced by the schematic letters. By the same token, what remains the same are the terms that determine the different moods - what we now refer to as quantifying and negating terms - and the general term of connection 'belongs to' (and of course word order), thus playing the role of mould. Alexander also comments on Aristotle's use of schematic letters, and clearly relates what takes the place of the schematic letters to the matter of arguments:

He uses letters in his exposition in order to indicate to us that the conclusions do not depend on the matter but on the figure, on the conjunction of the premises and on the moods. For so-and-so is deduced syllogistically not because the matter is of such-and-such a kind but because the combination is so-and-so. The letters, then, show that the conclusion will be such-and-such universally, always, and for every assumption. (Alexander of Aphrodisias, in Apr 53.28-54.2, translation p. 116)

Alexander does not explicitly say (here) what, in a syllogism, corresponds to its form, but by relating the use of schematic letters in the Aristotelian argument-schemata to indifference to specific matter, it is but a small step to conclude that the matter of the argument is referred to or simply is what is replaced by the schematic letters (the terms), while its form corresponds to the remaining terms. However, the remaining terms determine not the figure but rather the mood of a syllogism. Hence, while the Ancient Commentators usually say that the figure of a syllogism, not its mood, corresponds to its form, associations of the form versus matter distinction to the use of schematic letters seem to suggest that the mood of a syllogism, not its figure, would correspond to its form (more on this below). In other words, a certain ambiguity on how the form of a syllogism is to be understood is already present in these early texts.

In a similar passage, Alexander stresses that preservation of validity under different 'material instances' is precisely what makes syllogistic arguments reliable.

Combinations are called syllogistic and reliable if they do not alter together with differences in the matter - i.e. if they do not deduce and prove different things at different times, but always and in every material instance preserve one and the same form in the conclusion. Combinations which change and alter configuration together with the matter and acquire different and conflicting conclusions at

\footnotetext{
34 The term used by Aristotle for syllogistic figures in Greek is schema, which is indeed lexically very close to the usual term for form, eidos.
} 
different times, are non-syllogistic and unreliable. (Alexander of Aphrodisias, in Apr 52.20-24, translation p. 114)

Here is another passage which seems to attribute the validity of logical arguments and operations in general to their form rather than to their matter:

For conversions - and in general deductions in the figures - do not depend, as I have already said, on the peculiarities of the matter (which is different in different cases), but on the nature of the figures themselves. That is why proofs in their case are universal. (Alexander of Aphrodisiasin Apr 35.6-9, translation p. 91).

A few centuries later, Ammonius is more cautious in his deployment of hylomorphic concepts regarding arguments, speaking of analogy instead of outright identification:

In every syllogism there is something analogous to matter and something analogous to form. Analogous to matter are the objects (pragmata) themselves by way of which the syllogism is combined, and analogous to form are the figures. (Ammonius, in Apr 4.9-11, translation in Barnes 1990, 41).

Here, what seems to correspond to the matter of a syllogism are the things themselves, the objects named in the syllogism. Properly speaking, Ammonius is not saying that the terms signifying these objects in the argument ${ }^{35}$ are (analogous to) the matter of the argument, but rather that they signify (refer to, denote) this matter. But again it is but a small step to view these very terms as constituting the matter of the argument.

Besides commentaries on the Prior Analytics, other important loci for the application of the form versus matter framework to arguments were commentaries on the Sophistical Refutations. Ebbesen (1981a, 95-105) narrates the story of the transmission of this tradition from later Greek sources to the medieval Latin tradition. Briefly put, in the context of the doctrine of fallacies (which is the subject-matter of the Sophistical Refutations), the issue of the different ways in which an argument can be defective arises naturally. In effect, one of the divisions offered in the later ancient tradition, and one which remained influential throughout the Latin Middle Ages, was that between arguments that are formally defective and those that are materially defective. Formally defective arguments would be those that do not display a valid (syllogistic) mood, while materially defective arguments are those with false premises (thus echoing Aristotle's own remark that the premises are the matter to the conclusion). Ebbesen (1981a, 97) adds that "the distinction between materially and formally defective arguments, though a stock item of the commentaries, never became an organic part of the teaching of sophistic." Nevertheless, these were certainly significant applications of the form versus matter distinction to arguments, in particular insofar as the tradition seems to have passed on to the later Latin Middle Ages when the Sophistical Refutations and its commentaries were rediscovered in the twelfth and thirteenth centuries.

\footnotetext{
35 The medievals referred to these terms as categorematic terms, terms having a signification in themselves, while syncategorematic terms such as 'is', 'every', 'no' do not signify anything by themselves-a distinction already noted by Aristotle in De Int. 16b24-25.
} 
In short, taking Aristotle's mould analogy and his use of schematic letters in syllogistic as their starting point, the Ancient Commentators formulated an account of the form and matter of arguments that is clearly the first step towards LHAWKI. The Commentators tend to speak of figures as corresponding to the forms of arguments, but along the way between them and the Latin fourteenth century, the form of syllogisms became increasingly associated to syllogistic moods; more generally, the form of arguments became associated to schemata (syllogistic figures are not schemata in the modern sense, but syllogistic moods are). In the only still extant twelfth century Latin commentary on the Prior Analytics, dubbed Anonymus Aurelianensis III by S. Ebbesen (fragments of which are edited in Ebbesen 1981b), the form of a syllogism is said to be understood in two ways, following the typical medieval technique of distinguishing different senses of the same term ${ }^{36}$ :

Furthermore, the form of a syllogism is understood in two ways, namely the disposition of the terms, which is called its figure, and the disposition of the sentences, which is said to be its mood. (Latin in Ebbesen 1981b, 14, my translation)

And just as the form of syllogisms is understood in two ways, so is [their] matter understood in two ways, namely terms and sentences. (Latin in Ebbesen 1981b, 15 , my translation)

Whether the form of a syllogism corresponds to its figure or to its mood is a crucial point, as the latter presupposes a partition of the vocabulary into two sorts, while the former does not. In the form-as-figure case, we seem to have a notion of form as spatial structure, as figures simply codify the relative position of the terms within the syllogism; thus cast, the form versus matter opposition is not essentially mereological. In the form-as-mood case, however, the form-matter distinction is clearly mereological; the mood of a syllogism is also defined by the relative positions of its terms, to be sure, but it is crucially determined by a certain subset of its vocabulary, namely words such as 'every', 'some', 'is' and 'not'. Hence, the view that the form of a syllogism corresponds to its mood is already a (germinal) version of (mereological) LHAWKI, minus the demarcational role attributed to the formal vocabulary.

Indeed - and this was the second step towards LHAWKI-in subsequent developments the view that the form of a syllogism (more generally, a particular subset of an argument's vocabulary) pertains to its mood became increasingly prevailing. For reasons of space, I cannot spell out all the details of these developments, but the crucial steps were: Boethius' transmission of the gist of the Commentators' logical hylomorphism to the Latin world (but not of its terminology); Abelard's distinction between perfect and imperfect inferences, the former owing their validity to their 'construction' alone (again no use of explicit hylomorphical terminology); the Latin rediscovery of the Aristotelian corpus (including the Physics and the Metaphysics) and of the Ancient Commentators' tradition in the twelfth and thirteenth centuries ${ }^{37}$;

\footnotetext{
36 Robert Kilwardby (see next footnote) makes a similar suggestion-see Thom $(2007,57)$.

37 An interesting aspect of this rediscovery was the tendency in thirteenth century Latin authors to project Aristotelian metaphysics into logical analysis, something that Aristotle himself never did, and in fact even going beyond the use of metaphysical concepts in logic that one finds in the Ancient Commentators.
} 
the consolidation of the notions of formal and material consequence in the fourteenth century with Ockham, Buridan, Billingham and others. The crucial point is that, by the fourteenth century, LHAWKI was already almost fully mature. At that point, the notion of the form of an argument was not yet used for demarcational purposes, but the idea of the form of an argument as pertaining to a particular subset of its vocabulary (in a given disposition) was already fully developed, as can be perceived in the following passages by the fourteenth century author John Buridan:

'Formal' consequence means that [the consequence] holds for all terms, retaining the form common to all. Or, if you want to express it according to the proper force of discourse, a formal consequence is that which, for every proposition similar in form which might be formed, it would be a good consequence, such as 'some A is B; thus some B is A'. 38 (Buridan 1976, 22/23 (5-9), my emphasis and translation)

I say that, in the previous considerations (as we are speaking here of matter and form), we understand by the 'matter' of the proposition or consequentia the purely categorical terms, i.e. subjects and predicates, omitting the syncategorematic terms that enclose them and through which they are conjoined or negated or distributed or forced to a certain mode of supposition. All the rest, we say, pertains to the form. (Buridan 1976, 30 (7-12), my translation)

In other words, for Buridan the form of an argument pertains to its syncategorematic terms in a certain disposition, while its matter pertains to its categorematic terms. Buridan's notion of formal consequence is thoroughly substitutional, but he does not endorse a proto-form of LHAWKI because he does not view the validity of formal consequences as grounded in their form (for an account of Buridan's notion of consequence, see Dutilh Novaes 2005). ${ }^{39}$ Nevertheless, there is a continuous historical line from the Latin fourteenth century up to us, i.e. constant applications of the form versus matter distinction to arguments resulting in a (strict) partition of the vocabulary into terms belonging to form and terms belonging to matter. This general procedure tended to receive bad publicity in the Renaissance and early modern period, but in practice it continued to be used and applied. Via Leibniz and Kant, it made its way to Bolzano, and then on to Tarski. In fact, the general idea of the form of an argument as related to a subset of its terminology remained in the background throughout this

\section{Footnote 37 continued}

This trend is exemplified in particular by Robert Kilwardby-see Thom (2007). Here is an important passage for our purposes from Kilwardby's commentary on the Prior Analytics: "A syllogism, since it is a certain composite, ought to be composed of matter and form. Hence, if it is deficient in either, it will not be a syllogism" (quoted in Thom 2007, 47, his translation). Kilwardby clearly felt that it was important to justify why it makes sense to speak of the form and matter of a syllogism.

38 The Latin text as transcribed by Hubien actually has the following: "what is A is B, therefore what is B is A" ("quod est A est B; ergo quod est B est A"), which is obviously an invalid consequence-schema. This is indeed what one usually finds in the available manuscripts of the text, but it is probably a scribal error from an earlier manuscript (it is unlikely that Buridan himself would have made such a blunt mistake).

39 Moreover, Buridan acknowledges that the categorematic versus syncategorematic partition of the vocabulary is not a straightforward, clear-cut matter. For example, he recognizes the existence of 'limit cases', such as the verbs 'to see', 'to want', 'to know', which perform functions relevant to both the form and the matter of a sentence. See Buridan (2001, 4.3.8.4). 
period. Hence, it seems evident that the tradition discussed in Sect. 1 above is fully embedded in the historical developments just described here.

The last crucial step towards LHAWKI as characterized in Sect. 1 takes place with Kant: he is the first to have attempted to demarcate logic by means of its formality. Even though Kant himself did not rely on the substitutional-schematic notion of 'formal' for demarcational purposes, this is precisely what most of the subsequent tradition attempted to do, meshing the mereological hylomorphism as applied to arguments that had been available at least since the Latin Middle Ages with the Kantian demarcating enterprise. ${ }^{40}$ It is precisely this combination that gave birth to LHAWKI, and in particular to the problem of demarcating logical constants. I shall not review the historical details of the Kantian demarcational enterprise and the extent to which it still seems to underpin many of the currently widespread views on logic, as all this is aptly described in (MacFarlane 2000). For my purposes here, it is sufficient to stress that, in developments prior to Kant, the concern with demarcating logic from other disciplines was virtually absent, and that the scope of the themes and topics treated under the heading of logic was quite broad. (Just to illustrate the point, notice that Buridan distinguishes formal from material consequences by means of a substitutivity criterion, but he does not ban material consequences from the realm of logic.)

\section{Reassessing logical hylomorphism}

We now tend to assume that applying the form versus matter distinction to arguments in particular and to logic in general is a perfectly reasonable, natural thing to do; in fact, it would be difficult for most of us even to conceive the nature of logic in any other terms. In other words, we take the application of this distinction to logic for granted-we take logical hylomorphism for granted. But as the overview of the history of applications of this distinction to logic in Sect. 2 suggests, there are good historical reasons not to take logical hylomorphism for granted, as each step towards the development of LHAWKI involved substantive assumptions. There are also systematic, conceptual reasons:

- Are arguments the kind of things it makes sense to speak of in hylomorphic terms? For Aristotle, typically the concepts of form and matter are applied to substances, i.e. to wholes but not to heaps. But are arguments wholes or heaps? They are clearly not substances as an individual man or any of the items to which the form versus matter distinction is typically applied by Aristotle. This does not mean that applications of the distinction to arguments are automatically unwarranted, but it does seem that a justification would be required to ground these applications of hylomorphism to such 'unusual' objects such as arguments. ${ }^{41}$

- Are we justified in importing presuppositions and expectations pertaining to metaphysical hylomorphism into logical hylomorphism? Within metaphysical

\footnotetext{
40 I am not entirely sure as to when, after Kant, the Kantian demarcational project enlisted the age-old distinction between the form and the matter of an argument based on a partition of the vocabulary for its demarcating purposes.

41 An answer to this question would require an analysis of the metaphysics of arguments. What are arguments made of? Are they artifacts crafted by people for certain purposes, just as hammers and toothbrushes? Or do they have some sort of independent existence?
} 
hylomorphism, there is the presupposition of a sharp, factual distinction/demarcation between the form and the matter of a thing, as these two aspects of a thing are seen as being of different ontological kinds. Moreover, there is usually (though not always) the presupposition of uniqueness of form for each thing. We have seen that LHAWKI relies on the assumption of uniqueness of form and of a principled separation of form from matter. If it makes sense at all to apply the form versus matter distinction to arguments, should it come accompanied by these typically metaphysical presuppositions? (Notice however that within LHAWKI these assumptions are related to specific theses tacitly or explicitly held about logic, and are thus not only projections imported from the metaphysical context.)

- Should the separation between the form and the matter of arguments be used to demarcate the realm of logic? Does it make sense to define the scope and nature of logic exclusively in terms of a particular class of terms/notions? It is as if 'the class of logical notions' were a natural kind, having something like a Platonic independent existence and defining 'from above' the scope and nature of logic. The form versus matter partition would be an ontic matter, previously settled, and our task as logicians and philosophers of logic would be that of discovering the exact boundaries of this pre-existing natural kind. As such, these views clearly entail a strong form of Platonism about logic, which seems a rather costly position to hold.

These are issues that proponents of logical hylomorphism as a demarcating criterion, in particular related to the schematic notion of the formal and to the idea of a privileged class of logical constants, must address in order to provide a philosophically solid basis for LHAWKI. For the moment, none of them seems to have been properly tackled, and the extreme difficulty in establishing a principled criterion defining the class of logical notions/logical constants suggests that there is no such thing as the natural kind 'logical constants' - or else that, even if there is such a class, we humans are not cognitively well equipped to discover its boundaries.

It might be objected (as has been objected by an anonymous referee) that contemporary philosophers and logicians are not likely to recognize themselves and many of their views as ultimately stemming from the Aristotelian and Kantian concepts and concerns described in Sect. 2. That is probably true, but this is at least to some extent related to an insufficient knowledge of the history of their discipline. ${ }^{42}$ More importantly, it might be thought that, even if there is some murky, remote historical connection, it is by now too frail to be of philosophical interest. Now, the point of the present contribution is to invite these contemporary philosophers and logicians to reflect on and reassess the tacit assumptions underlying many of their views about logic, and it seems to me that paying attention to the historical development of these assumptions is a fruitful reflection-trigger. I believe that the connections that emerge between the 'textbook' view discussed in Sect. 1 and the historical developments discussed in Sect. 2 speak for themselves. More generally, the need for a broad re-evaluation of

\footnotetext{
42 MacFarlane (2000) has done a great job at showing how deeply (and usually unwittingly) influenced by the Kantian conception of logic we still are. Admittedly, the connection with the Ancient Commentators on Aristotle is more remote and would deserve a more thorough examination than what I provide here, but at the very least the present analysis suggests that it is a hypothesis worth taking into account.
} 
the assumptions underpinning logical hylomorphism as we know it is evident, and a deeper knowledge of the history of the distinction with respect to logic seems like an appropriate approach (albeit of course not the only one) for this end.

So here is one possible diagnose of the situation: uses of the substitutional-schematic technique to formulate accounts of the very grounds for the validity of arguments and of the scope of logic rest on the mistake of confusing a particular method used in logic with its very subject-matter. ${ }^{43}$ The quasi-metaphysical question of the ultimate grounds for argument validity simply cannot be resolved by an appeal to the method of working with schemata; this method has been extensively used since Aristotle as a means to capture interesting patterns among valid arguments, but not (or rarely) as an explanation for the very phenomenon of argument validity. ${ }^{44}$ The substitutional technique of allowing for some terms to remain fixed while others are replaced by schematic letters, yielding schemata, pertains to the practice of logic; the quest for that in virtue of which valid arguments are valid pertains to the philosophy of logic. Of course, there is no reason why these two must be strictly kept apart: the practice of logic can certainly benefit from philosophical insights, and the philosophy of logic should deal for as much as possible with the actual practices of logicians. Nevertheless, the questions being asked in each domain are of a different nature: the logician wants tools that allow her to study systematically the (logical) properties of valid arguments; the philosopher of logic raises questions such as "what is logic?", "what makes a valid argument valid?" etc.

My proposal is thus not to get rid of the schematic-substitutional technique-it has been crucial for much of the progress made in logic so far, and it still seems to have much to deliver-but rather to give up on the idea that this technique by itself can resolve deeper philosophical issues such as the demarcation of logic. If logic as a discipline can be demarcated at all, it seems that this cannot be done by simply isolating a particular subset of notions or concepts as quintessentially logical by means of a (sharp, principled) partition of the vocabulary. This being said, I surely do not wish to imply that philosophical questions such as the grounds for the validity of arguments and the nature of logic should be dropped altogether. But these questions must be addressed in their own terms, i.e. philosophical terms.

\footnotetext{
43 I guess this claim really makes me be a Debunker in MacFarlane's sense (2009, Sect. 8): "They do not dispute that logicians have traditionally concerned themselves with argument forms in which a limited number of expressions occur essentially. What they deny is that these expressions and argument forms define the subject matter of logic. On their view, logic is concerned with validity simpliciter, not just validity that holds in virtue of a limited set of "logical forms." The logician's method for studying validity is to classify arguments by their forms, but these forms (and the logical constants that in part define them) are logic's tools, not its subject matter."

44 One of the few pre-twentieth century, worked-out accounts of the validity of some arguments in terms of their construction is Abelard's discussion of the validity of perfect inferences in the Dialectica. This being said, Abelard does not claim that imperfect inferences are not valid: rather, they are valid, but their validity rests on 'the nature of things'. Aristotle himself offers a very general definition of a valid argument, in terms of the conclusion following 'of necessity' from the premises-see Prior Analytics, 24b19-24.
} 
So here are some of the plausible positions one could hold on the relation between hylomorphism and $\operatorname{logic}{ }^{45}$ :

- The application of hylomorphism to logic is infelicitous; arguments and other logical objects are simply not the kind of things having 'form' and 'matter'. Applying the distinction to logic entails the risk of an undue projection of expectations and presuppositions (such as that there is a sharp, factual distinction between form and matter and that there is one unique form for each thing) that only distorts our understanding of logic. This position amounts to a rejection of all of the eight theses formulated in Sect. 1 as characterizing LHAWKI.

- (Mereological) hylomorphism can be applied to logic, but only as a metaphor that should not be pushed over its limits; as well said by Ammonius, in an argument there is something analogous to form and something analogous to matter. If it is but an analogy, then there is no need to establish a principled, sharp demarcation between the form and the matter of logical objects, as this can be a matter of pragmatic choice given the circumstances. It would seem that this metaphorical/analogical form of logical hylomorphism was the one actually endorsed by logicians and philosophers in most of its history, certainly up until demarcational concerns came into the picture with Kant. In particular, it served as support for the development of the schematic-substitutional technique that was decisive for the development of logic as a discipline. (But notice that Aristotle himself uses this technique in the Prior Analytics, in particular in order to prove the invalidity of some syllogistic moods, so the technique itself predates logical hylomorphism.) This position amounts to a rejection of some of the eight theses formulated in Sect. 1 as characterizing LHAWKI, in particular thesis 4 and thesis 8 (possibly 7 as well).

- This particular form of mereological logical hylomorphism does not work, but perhaps a different understanding of hylomorphism may provide a more illuminating account of logic from a hylomorphic perspective. In particular, the non-mereological, functionalist hylomorphism that emerges from Aristotle's own writings may provide a fruitful framework to think about logic in hylomorphic terms. On a functionalist approach to hylomorphism, form corresponds to a functional principle of unity, i.e. that in virtue of which the object in question fulfils the function(s) that characterize(s) it as an object. This position amounts to a rejection of many of the eight theses formulated in Sect. 1 as characterizing LHAWKI, but an endorsement of theses 1, 4 and 5 .

On a functionalist approach to logic, the first question to be asked is: What is the function of a valid argument? What is it that differentiates a valid from an invalid argument? A functionalist approach to logic may shift the focus away from the issue of a partition of the vocabulary into logical and non-logical expressions in favor of an analysis of deeper issues; arguably, LHAWKI severely misidentifies the real core of the phenomenon of argument validity. Of course, thus formulated, a functionalist

\footnotetext{
45 I do not discuss here the Kantian blend of logical hylomorphism, according to which logic deals with the pure forms of the understanding, as a proper analysis of this position would require a significant amount of space. Suffice it to say that I believe it to be equally problematic, albeit for different reasons.
} 
approach to logic is not a framework that delivers all the answers, but it may be a framework that allows for the formulation of the right questions.

At any rate, what the present analysis suggests is that the particular form of logical hylomorphism that we seem to take for granted as the only natural one, LHAWKI, is just one possible, and in fact a rather dubious, outcome of the combination of hylomorphism and logic. There are other options available: outright rejection of the explanatory power of the hylomorphic framework when it comes to logic; an analogical, metaphorical application of the notions of form and matter to logical objects, with no further theoretical commitments or projections; taking a different notion of hylomorphism as a starting point for the development of a logical hylomorphism. (There may be other options as well, I do not claim to be exhaustive here.) These three options all seem to avoid some of the traps that LHAWKI finds itself struggling with, in particular the issue of demarcating in a sharp, principled manner the class of logical constants as a means to define the very scope and nature of logic. It is the endorsement of all eight of the theses formulated in Sect. 1 that seems to lead to such issues. This being said, I am not claiming that LHAWKI cannot be salvaged; perhaps it can, but this would require that the issues raised throughout the paper be properly addressed. So it seems to me that the ball is now on the court of the advocates of LHAWKI.

\section{Conclusion}

The historical goal of the paper was to unearth the historical roots of LHAWKI. In particular, I have argued that the main steps of these developments were: the application of the form versus matter distinction to arguments with the Ancient Commentators; the association of the schemata underlying arguments to their form in later developments (in particular in the Latin Middle Ages); the co-opting of the notion of form in logic for demarcational purposes with and after Kant.

However, the main aim of the paper was systematic, namely that of reflecting on the propriety of applying the form versus matter distinction to logical objects such as arguments, and to logic in general. The conclusion to be drawn is that the particular form of logical hylomorphism that appears to be widely but tacitly endorsed, LHAWKI, is not nearly as innocent and straightforward as most of us seem to think. It is based on substantive and often contentious assumptions; it fails to address some important foundational issues (e.g. are logical objects the kind of objects having form and matter?); and it yields thorny issues that seem to arise from undue conceptual projections (in particular the issue of demarcating the class of logical constants in a sharp, principled way). With this paper, I hope to provide further arguments supporting the search for an alternative philosophical account of logic, one where the usual issues that emerge within LHAWKI may be avoided. In particular, I have argued that a shift of focus away from the issue of demarcating the logical from the non-logical may be beneficial for our understanding of logic in general; it seems that worrying about the borders makes us lose sight of the core.

Acknowledgements Thanks to Stephen Read, Arianna Betti, Anthony Booth and Eric Schliesser for helpful comments on earlier drafts. 
Open Access This article is distributed under the terms of the Creative Commons Attribution Noncommercial License which permits any noncommercial use, distribution, and reproduction in any medium, provided the original author(s) and source are credited.

\section{References}

Alexander of Aphrodisias. (1991). On Aristotle's prior analytics 1.1-7 (J. Barnes, S. Bobzien, K. Flannery, K. Ierodiakonou, Trans.). London: Duckworth.

Barnes, J. (1990). Logical form and logical matter. In A. Alberti (Ed.), Logica, Mente e Persona. Florence: Leo S. Olschki.

Barnes, J. (2007). Truth etc. Oxford: Oxford University Press.

Bonnay, D. (2008). Logicality and invariance. Bulletin of Symbolic Logic, 14(1), $29-68$.

Buridan, J. (1976). Tractatus de Consequentiis. H. Hubien (Ed.). Louvain: Publications Universitaires. Buridan, J. (2001). Summulae de Dialectica (G. Klima, Trans.). New Haven: Yale University Press.

Cohen, S. M. (1992). Hylomorphism and functionalism. In M. C. Nussbaum \& A. O. Rorty (Eds.), Essays on Aristotle's De Anima (pp. 57-73). Oxford: Oxford University Press.

Corcoran, J. (2008). Schemata. In E. Zalta (Ed.), Stanford encyclopedia of philosophy. http://plato. stanford.edu/entries/schema/.

Dutilh Novaes, C. (2005). Buridan's consequentia: Consequence and inference within a token-based semantics. History and Philosophy of Logic, 26(4), 277-297.

Ebbesen, S. (1981a). Commentators and commentaries on Aristotle's Sophistici elenchi, Vol. I (the Greek tradition). Leiden: E. J. Brill.

Ebbesen, S. (1981b). Analyzing syllogisms or anonymus Aurelianensis III-the (presumably) earliest extant Latin commentary on the prior analytics, and its Greek model. CIMAGL, 37, 1-20.

Etchemendy, J. (1990). The concept of logical consequence. Cambridge, MA: Harvard University Press.

Etchemendy, J. (2008). Reflections on consequence. In D. Patterson (Ed.), New essays on Tarski and philosophy (pp. 263-299). Oxford: Oxford University Press.

Feferman, S. (1999). Logic, logics, and logicism. Notre Dame Journal of Formal Logic, 40(1), 31-54.

Gamut, L. T. F. (1991). Logic, language, and meaning-Vol. 1: Introduction to logic. Chicago: University of Chicago Press.

Gomez-Torrente, M. (2002). The problem of logical constants. Bulletin of Symbolic Logic, 8, 1-37.

Goodman, N. (1955). Fact, fiction, and forecast. Cambridge, MA: Harvard University Press.

Johnston, M. (2006). Hylomorphism. Journal of Philosophy, 103(12), 652-699.

Koslicki, K. (2006). Aristotle's mereology and the status of forms. Journal of Philosophy, 103(12), $715-736$.

Lagerlund, H. (2004). Medieval theories of the syllogism. In E. Zalta (Ed.), Stanford encyclopedia of philosophy. http://plato.stanford.edu/entries/medieval-syllogism/.

MacFarlane, J. (2000), What does it mean to say that logic is formal? PhD Dissertation, University of Pittsburgh. http://johnmacfarlane.net/diss.html.

MacFarlane, J. (2009), Logical constants. In E. Zalta (Ed.), Stanford encyclopedia of philosophy. http:// plato.stanford.edu/entries/logical-constants/.

McCarthy, T. (1981). The idea of a logical constant. Journal of Philosophy, 78, 499-523.

Prior, A. (Ed.). (1976). What is logic? In Papers on logic and ethics (pp. 122-129). London: Duckworth.

Rawls, J. (1971). A theory of justice, 2nd edition 1999. Cambridge, MA: Harvard University Press.

Read, S. (1995). Thinking about logic. Oxford: Oxford University Press.

Sher, G. (2008). Tarski's thesis. In D. Patterson (Ed.), New essays on Tarski and philosophy (pp. 300-339). Oxford: Oxford University Press.

Simons, P. (Ed.). (1992). Bolzano, Tarski, and the limits of logic. In Philosophy and logic in central Europe from Bolzano to Tarski (pp. 13-40). Dordrecht: Kluwer.

Tarski, A. (1936/2002). On the concept of following logically. History and Philosophy of Logic, 23, $155-196$.

Tarski, A. (1966/1986). What are logical notions? History and Philosophy of Logic, 7, 143-154.

Thom, P. (2007). Logic and ontology in the syllogistic of Robert Kilwardby. Leiden: Brill.

van Benthem, J. (1989). Logical constants across varying types. Notre Dame Journal of Formal Logic, 30, 315-342.

Wagner, S. J. (1987). The rationalist conception of logic. Notre Dame Journal of Formal Logic, 28, 3-35.

Warmbrod, K. (1999). Logical constants. Mind, 108, 503-538. 